Supporting Information for

\title{
Adjusting Emission Wavelength by Tuning the Intermolecular Distance in Charge-Regulated Supramolecular Assemblies
}

Hui Li, Ting-Zheng Xie, Zihao Liang, Yidan Shen, Xinyu Sun, Yuqing Yang and Tianbo Liu*

Correspondence to: tliu@uakron.edu

This PDF file includes:

Materials and Methods

Supplementary Text

Figures. S1 to S9

Tables S1, S2 


\section{Materials and Methods}

\section{Materials:}

Reagents and solvents were purchased from Sigma-Aldrich and used without purification. 1,1,2,2-tetrakis(4-(pyridin-4-yl)phenyl)ethene, sodium isophthalate, sodium [1,1'-biphenyl]-4,4'dicarboxylate, $c i s-\mathrm{Pt}\left(\mathrm{PEt}_{3}\right)_{2}(\mathrm{OTf})_{2}$, cis- $\mathrm{Pt}\left(\mathrm{PEt}_{3}\right)_{2}\left(\mathrm{NO}_{3}\right)_{2}$, Cage 1.8OTf- and Cage 1.8 $\mathrm{NO}_{3}^{-}$were synthesized according to the reported procedures. ${ }^{1-2}$ Deionized water was purified by Milli-Q Direct-Q3 water purification system. The polytetrafluoroethylene (PTFE) filters were purchased from EMD Millipore Corporation.

\section{Methods:}

Sample preparation. Solid samples of cages were dissolved in good solvents, for example, acetonitrile, and were diluted to appropriate concentrations for characterization. Typically, $30 \mu \mathrm{L}$ concentrated solutions were titrated into $3.0 \mathrm{~mL}$ designed solvents for further study. The volume fraction of good solvents in the resulting solution system was controlled as $1 \%$. All solvents were dust-freed by using PTFE filters.

Static and Dynamic Laser Light Scattering (SLS and DLS). A commercial Brookhaven instrument laser light scattering spectrometer with a solid-state green laser was used for both SLS and DLS measurements. During the DLS experiments, the intensity-intensity time correlation function was measured by means of a BI-9000AT multi-channel digital correlator. The field correlation function $\left|g^{(1)}(\tau)\right|$ was analyzed by CONTIN method $^{3}$ to yield the normalized distribution function of the characteristic linewidth $G(\Gamma)$, which can be used to determine the apparent translational diffusion coefficient $D=\Gamma / q^{2}$. The average hydrodynamic radius $\left(R_{\mathrm{h}}\right)$ of the 
assemblies can be calculated by using the Stokes-Einstein equation: $R_{\mathrm{h}}=k T /(6 \pi \eta D)$ where $k$ is the Boltzmann constant, $\eta$ is the viscosity of the solvent, $T$ is the temperature. The size distribution of the nanoscale assemblies can be obtained by plotting $\Gamma G(\Gamma)$ vs. $R_{\mathrm{h}}$ that $\Gamma G(\Gamma)$ is related to the angular-dependent scattered intensity of particles. The temperature in the sample chamber $(25.0$ ${ }^{\circ} \mathrm{C}$ ) of the LLS instrument was controlled within $\pm 0.1{ }^{\circ} \mathrm{C}$. SLS measurement was carried out by changing the scattering angles from $30^{\circ}$ to $120^{\circ}$ consequently, at $2^{\circ}$ interval. The collected scattering intensities from different angles were further analyzed by a partial Zimm plot with the software provided by Brookhaven Instruments Inc. to get the radius of gyration $\left(R_{\mathrm{g}}\right)$ of the particles.

Transmission electron microscopy (TEM). The TEM images were taken on a JEOL JEM-1230 electron microscope operated at $120 \mathrm{kV}$ accelerating voltage. A digital CCD camera as the detector was used to take and process the images. The sample solution for LLS studies were directly used for TEM measurements.

UV-Vis and fluorescence. UV-Vis spectrum was measured on Agilent Technologies 8453 UVVis Spectrophotometer from Aligent Technologies and analyzed by UV-Vis ChemStation Software. Fluorescence spectra were obtained on a HORIBA Jobin Yvon NanoLog spectrometer with excitation wavelength $355 \mathrm{~nm}$.

\section{Supplementary Text}

$\underline{\text { Counterion Association Process vs. }} \underline{\text { Concentration }}$ 
Counterion association process show sample concentration dependence, and the relationship can be expressed as:

\section{Cage $\cdot$ counterions $\leftrightarrow$ Cage + counterions}

High cage concentration facilitates counterions associated onto cages and the effective charges of the cages will be low, which will lead to a small curvature and large size in chargeregulated blackberry-type assemblies. 

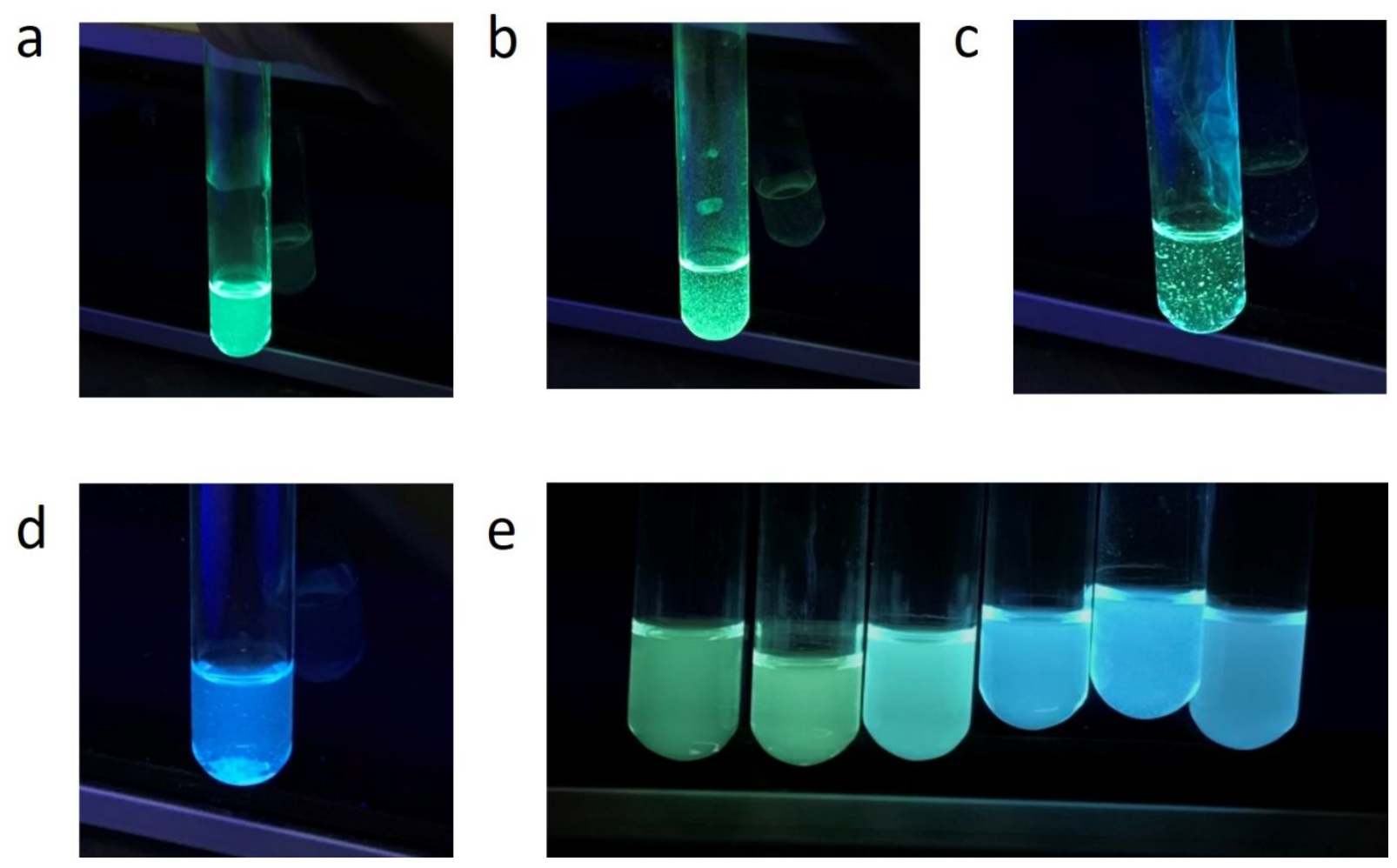

Figure. S1. The photographs of the aggregates from Cage 1 with nitrates as counterions from (a) toluene (b) hexane (c) 1,4-dioxane (d) the blackberry solutions and aggregates from tetrahydrofuran at different concentrations (from left to right, concentration increases). 


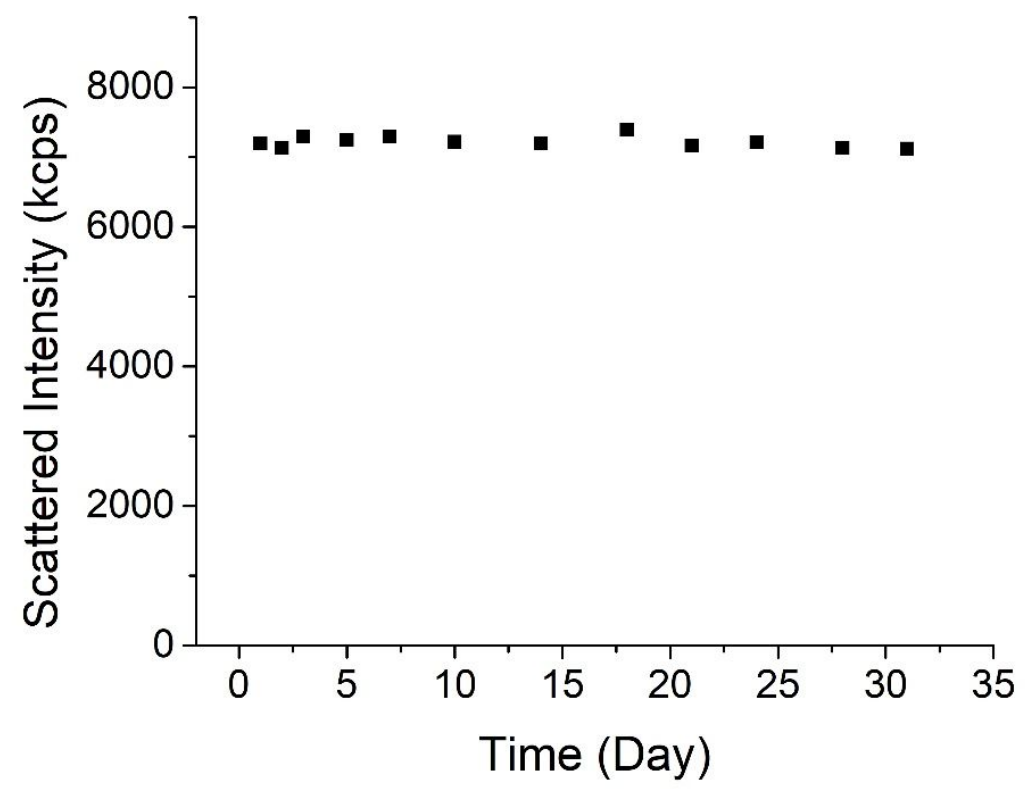

Figure. S2. Scattered intensities from the ethyl acetate (EA) solution of Cage 1 at $0.05 \mathrm{mg} / \mathrm{mL}$. The intensity kept at the same level within one month. 


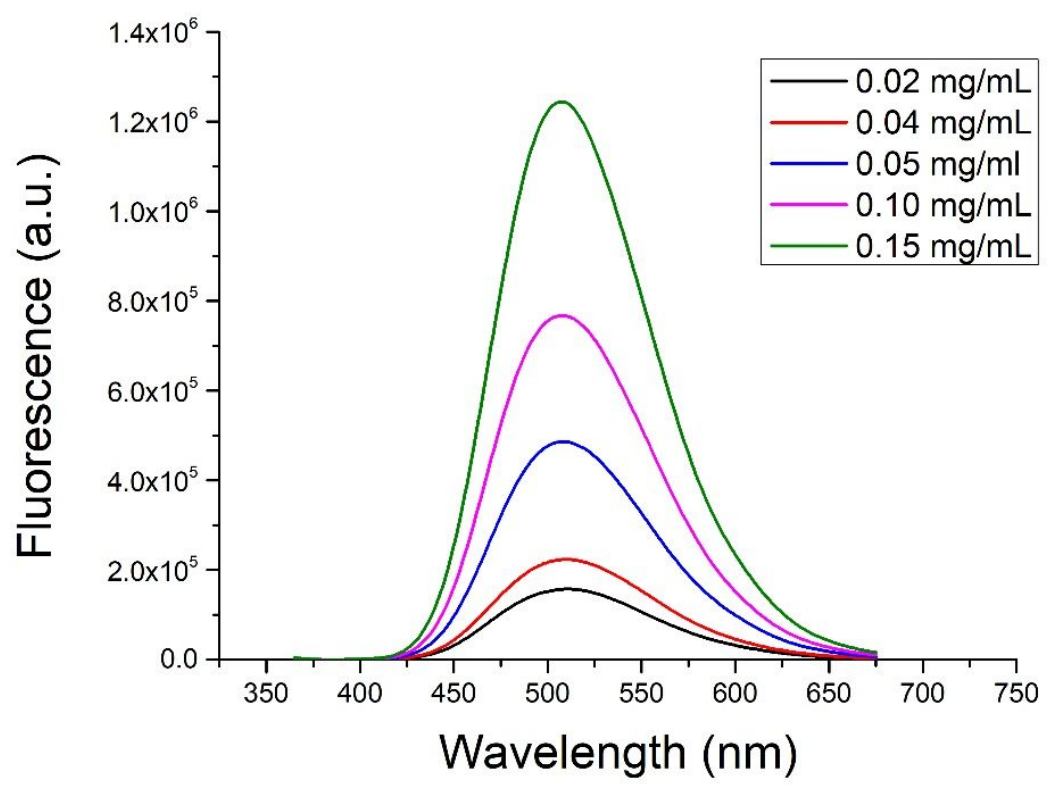

Figure. S3. Fluorescence spectra of Cage 1 blackberry-type assembly structures in ethyl acetate excited at $355 \mathrm{~nm}$. Concentrations: 0.02, 0.04, 0.05, $0.10 .015 \mathrm{mg} / \mathrm{mL}$. 


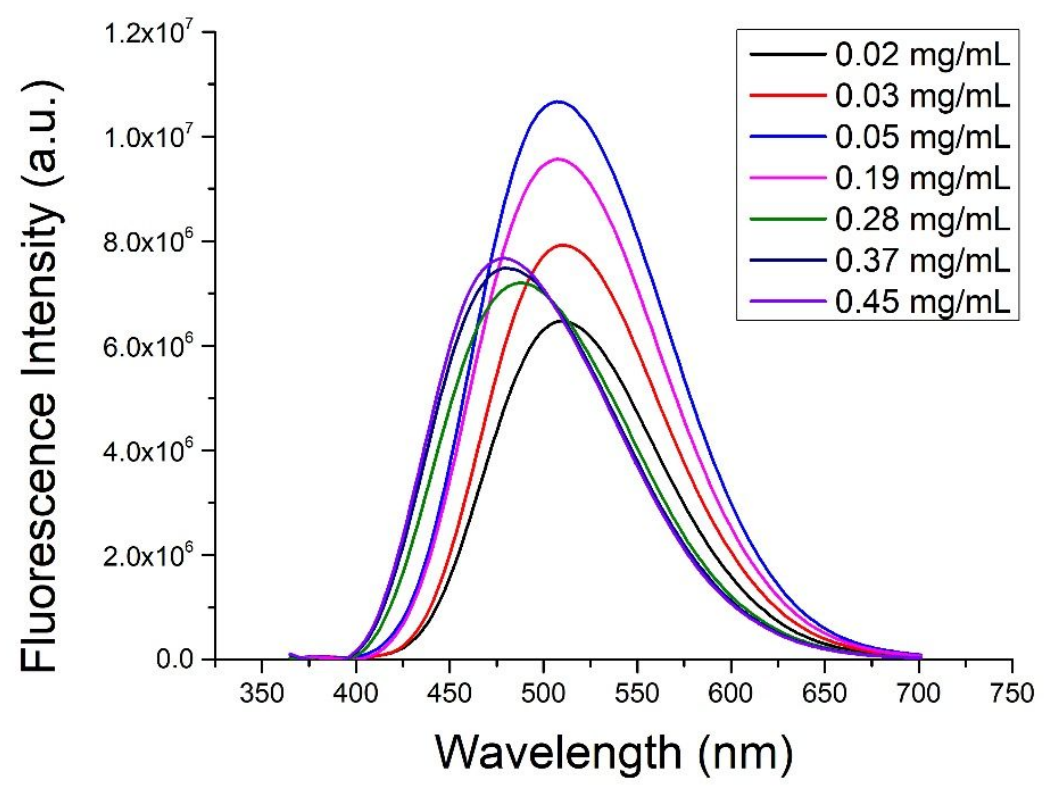

Figure. S4. Fluorescence spectra of Cage $1\left(\mathrm{NO}_{3}^{-}\right)$as blackberry structures and aggregates from THF solutions at different concentrations. 


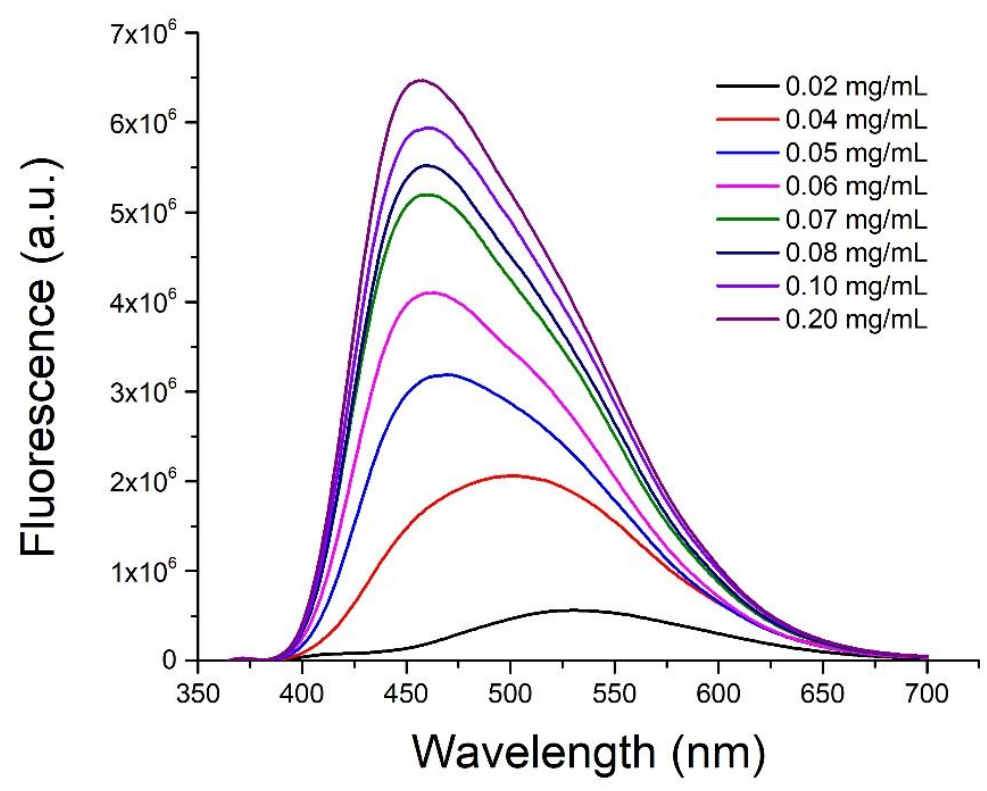

Figure. S5. Fluorescence spectra of Cage $1\left(\mathrm{OTf}^{-}\right)$as solutes, blackberry structures and aggregates from THF solutions at different concentrations. 


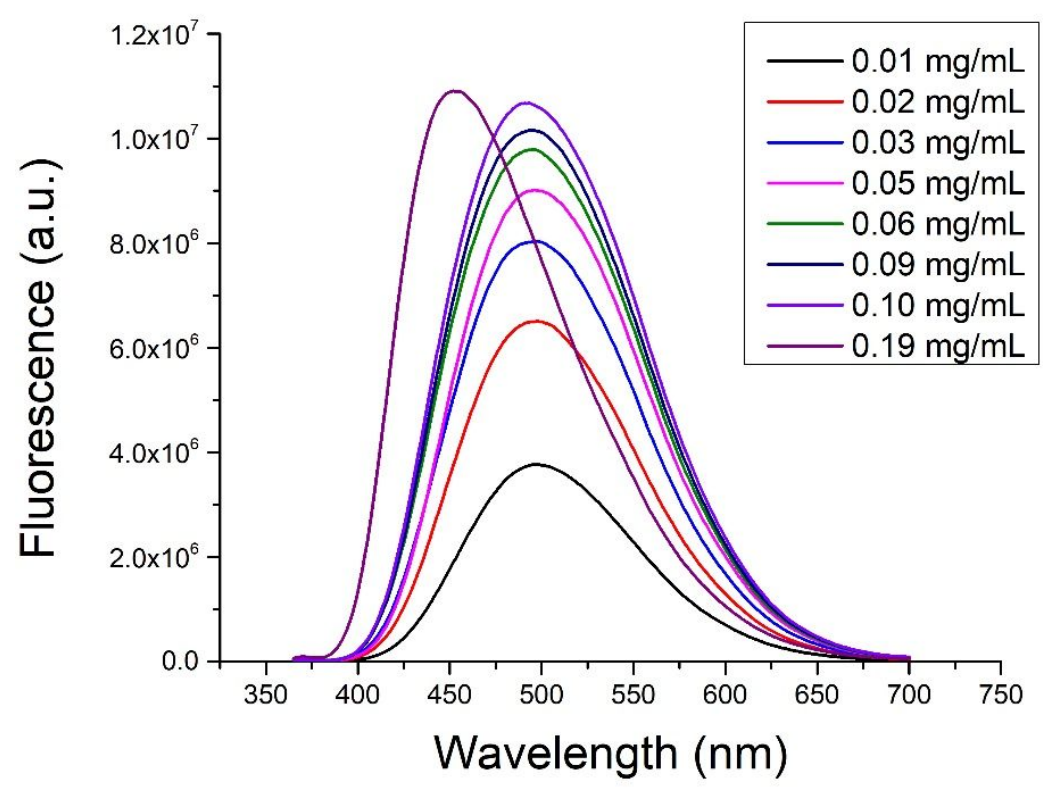

Figure. S6. Fluorescence spectra of Cage 1 (OTf-) in ethyl acetate solutions at different concentrations. 


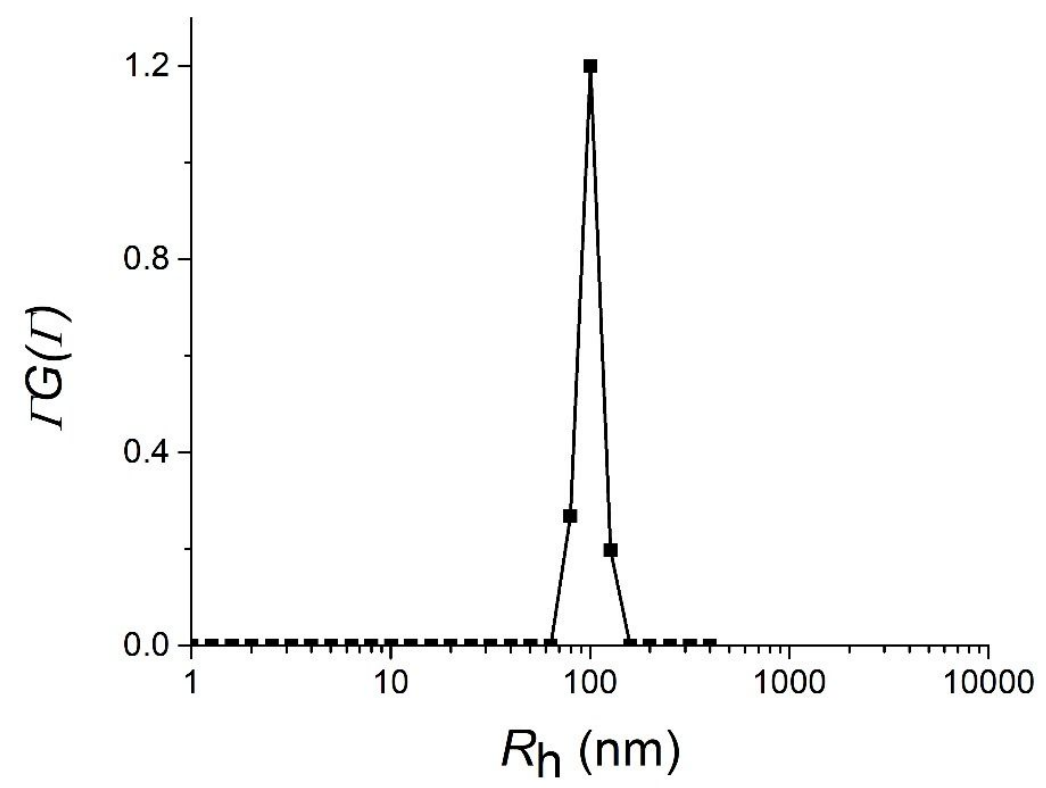

Figure. S7. $R_{\mathrm{h}}$ distribution of the blackberry structures from Cage 1 (with triflate anions) in 0.04 mg/mL THF solution. 
A

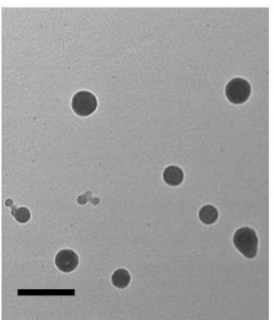

B

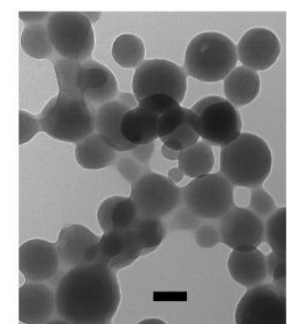

C

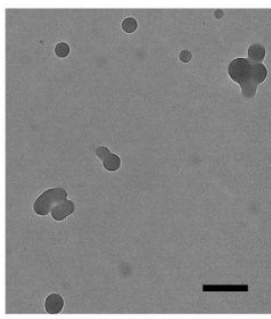

D

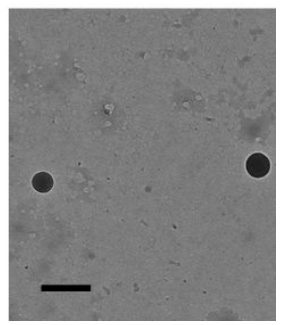

Figure. S8. TEM images of the blackberry structures of Cage 1 from ethyl acetate (with nitrate anions) and THF (with triflate anions) solutions. A, $0.02 \mathrm{mg} / \mathrm{mL}$ in ethyl acetate solution, length bar, $200 \mathrm{~nm} . \mathrm{B}, 0.05 \mathrm{mg} / \mathrm{mL}$ in ethyl acetate solution, length bar, $200 \mathrm{~nm} . \mathrm{C}, 0.15 \mathrm{mg} / \mathrm{mL}$ in ethyl acetate solution, length bar, $400 \mathrm{~nm}$. D, $0.04 \mathrm{mg} / \mathrm{mL}$ in THF solution, length bar, $400 \mathrm{~nm}$. 


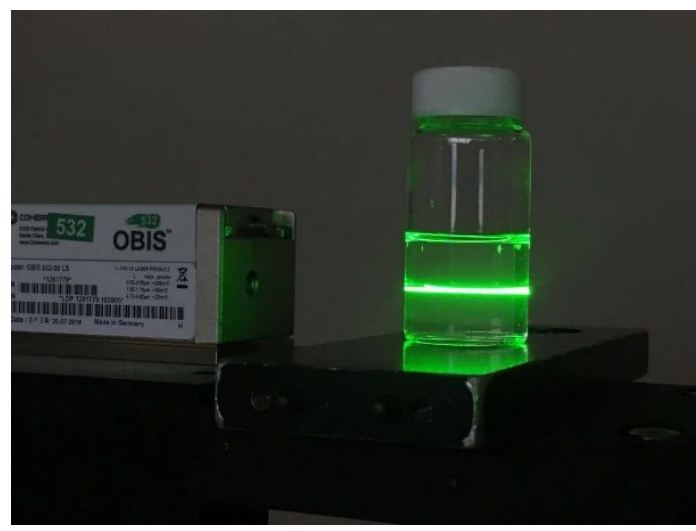

Figure. S9. Photograph showing Tyndall effect of the EA solution containing blackberry structures. 
Table S1. Scattered intensities of solvents and cage (with nitrate anions as counterions) solutions measured from static light scattering measurements.

\begin{tabular}{|c|c|c|c|}
\hline Solvent & $\mathrm{I}_{1}(\mathrm{kcps})$ & $\mathrm{I}_{2}(\mathrm{kcps})$ & $\begin{array}{c}\text { Dielectric } \\
\text { Constant }\left(\varepsilon_{\mathrm{r}}\right)\end{array}$ \\
\hline DMSO & 5.0 & 6.0 & 46.7 \\
\hline Acetonitrile & 3.0 & 4.7 & 37.5 \\
\hline DMF & 3.5 & 3.8 & 36.7 \\
\hline Methanol & 4.0 & 4.5 & 32.7 \\
\hline Acetone & 2.6 & 4.0 & 20.7 \\
\hline dichloromethane & 3.5 & 3.9 & 8.9 \\
\hline THF & 6.0 & 3760 & 7.6 \\
\hline Ethyl acetate & 4.2 & 7200 & 6.0 \\
\hline 1,4-dioxane & 3.8 & $>3000^{\mathrm{a}}$ & 2.3 \\
\hline Hexane & 5.7 & $>5000^{\mathrm{a}}$ & 1.9 \\
\hline
\end{tabular}

$I_{1}$, scattered intensity from dust-freed solvents. $I_{2}$, scattered intensity from cage solutions. Concentration of cage: $0.05 \mathrm{mg} / \mathrm{mL}$, room temperature. Kcps: thousand counts per second. ${ }^{\text {a }}$ cages precipitate from these solvents and the scattered intensities show fluctuations during the measurements. 
Table S2. The peak position of maximum emission of cages (with nitrate anions as counterions) in different solvents at $0.05 \mathrm{mg} / \mathrm{mL}$.

\begin{tabular}{|c|c|}
\hline Solvent & $\begin{array}{c}\text { Peak Center } \\
(\mathrm{nm})\end{array}$ \\
\hline Methanol & 552 \\
\hline Acetonitrile & 550 \\
\hline Acetone & 550 \\
\hline THF & 507 \\
\hline Ethyl acetate & 509 \\
\hline Hexane & 507 \\
\hline 1,4-dioxane & 500 \\
\hline
\end{tabular}

Cages precipitated from hexane, 1,4-dioxane at this concentration. For these systems, measurements were taken by using the aggregates and solvent mixture. 


\section{References:}

(1) Yan, X.; Cook, T. R.; Wang, P.; Huang, F.; Stang, P. J., Nature chemistry 2015, 7, 342.

(2) Li, H.; Xie, T.-Z.; Liang, Z.; Dahal, D.; Shen, Y.; Sun, X.; Yang, Y.; Pang, Y.; Liu, T., Chem. Commun. 2019, 55, 330-333.

(3) Provencher, S. W., Comput. Phys. Commun. 1982, 27, 229-242. 